\title{
Concussions and their consequences: current diagnosis, management and prevention
}

\author{
Charles H. Tator MD PhD
}

See also practice article by Purcell and colleagues on page 981 and at www.cmaj.ca/lookup/doi/10.1503/cmaj.120511

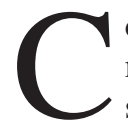

oncussion is the most common type of mild traumatic brain injury and can have serious consequences. Not just confined to high-profile athletes, concussions are frequent in all age groups and in a variety of settings, such as the work environment, motor vehicle crashes, sports and recreation, and falls at home among older people. Concussion is defined by the International Consensus Conference on Concussion in Sports as "a complex pathophysiological process affecting the brain, induced by biomechanical forces." Concussion is the preferred term because of its familiarity to the public. Since 2000, international expert panels have clarified the definition and modified the management of concussion; these changes have affected recommendations for return to work, school and sport for those experiencing a concussion. ${ }^{1.2}$

The importance of accurate and timely recognition and management stems from the consequences of misdiagnosis or faulty management that can lead to major disability or death, in both the short and long term. Second-impact syndrome occurs when a concussed person, especially a younger person, returns to play before complete recovery and sustains a second brain injury. However, malignant brain swelling can occur even without a second injury. ${ }^{3}$ Also, repeated concussions may cause delayed posttraumatic brain degeneration, leading to dementia and movement disorders similar to Alzheimer and Parkinson diseases. ${ }^{4}$ Thus, it is important for practitioners to know the current principles of recognition and management of concussions, including the physical, cognitive and emotional effects and the guidelines for return to play, work or school.

\section{Mechanism}

The exact mechanism of concussion is unknown. Axon tearing occurs in more severe brain injuries, but proof is lacking for this mechanism in concussion. It is more likely that concussion is due to rotational acceleration of the brain (jiggling of the brain) ${ }^{5}$ that produces a disordered metabolic cascade or biochemical injury such as altered metabolism of glucose or derangement of adenine nucleotides. ${ }^{6}$ It is still uncertain where in the brain concussion occurs, or the exact origin of the symptoms of acute concussion. It is now apparent that direct impact to the head is not required: concussion can occur with a blow to the chest, for example, that causes a whiplash effect on the brain. Whiplash of the neck and concussion frequently co-exist.?

\section{Risk factors}

The young brain is more susceptible to concussion than the adult brain and may require more time to recover. ${ }^{8}$ Also, it is now known that, after a concussion, there is greater susceptibility to sustaining another concussion and that subsequent concussions occur with less force and take longer to resolve. ${ }^{9} \mathrm{New}$ information indicates that females may be more susceptible to concussion than males ${ }^{10}$ and that there may be a genetic factor underlying susceptibility to concussion. ${ }^{11}$

\section{Ker POINTS}

- Major changes in the definition and management of concussions have occurred over the past decade.

- Concussions, especially repeated concussions, are now recognized as an important public health risk.

- Physicians play a key role in the diagnosis and management of concussions, especially in facilitating individualized timetables and activities for return to play, school and work.

- To prevent concussions and their consequences, physicians need to advise patients about safe practices and, when symptoms persist, advise whether to desist temporarily or permanently from activities with a high risk for concussion. 


\section{Diagnosis}

The diagnosis of concussion is not easy and requires a knowledgeable physician and a cooperative patient. In the absence of both, accurate diagnosis and management may not be possible. A concussed athlete may minimize or hide the symptoms or disregard management advice if he or she aims to avoid removal from play. ${ }^{12,13}$

Concussion is a diffuse injury without focal neurologic deficits such as pupillary dilation or limb weakness. The symptoms are usually subtle, such as dizziness and "seeing stars." The most frequent symptoms are headache, dizziness, nausea and imbalance. Loss of consciousness occurs in a minority of cases. The first concussion is usually mild, with full recovery within $7-14$ days. ${ }^{1}$

The diagnosis can be made only clinically because there is no proven biomarker based on imaging, blood tests or computerized neuropsychological screening tool. Conventional computed tomography (CT) and magnetic resonance imaging (MRI) scans almost always appear normal after concussions, even after repeated concussions. ${ }^{1}$ Numerous biomarkers of concussion are being investigated, including electrophysiology of brain interactions or connectivity, and evoked potential abnormalities. Several of the current investigations are based on MRI and include functional MRI, diffusion tensor imaging and MR spectroscopy. Serum and cerebrospinal fluid biomarkers are also being explored, especially those that might detect a genetic basis for susceptibility to concussion, but to date none has been proven. ${ }^{11}$ Several computerized neuropsychological tests are being promoted commercially as "baseline" tests; at best, they are an adjunct to clinical examination and cannot be used as stand-alone tests to diagnose concussion. ${ }^{14}$

\footnotetext{
Box 1: Protocol for graduated return to play*

1. No activity: Complete cognitive and physical rest

2. Light aerobic exercise: Walking, swimming, stationary cycling

3. Sport-specific exercise: Skating or running drills; no head-impact activities

4. Non-contact training drills: $\dagger$ More vigorous training drills

5. Full-contact practice: Normal training activities after medical clearance

6. Return to play: Normal game play

*When all symptoms have completely disappeared in one step, the next step is initiated.

tPhysician re-examination is recommended after step 4

before full-contact practice can be resumed.

Source: McCrory et al. ${ }^{1}$
}

A useful diagnostic aid for practitioners is the third edition of the Sport Concussion Assessment Tool (SCAT3, http://links.lww.com /JSM/A30), a clinical examination protocol that lists 22 possible symptoms of concussion. The presence of only 1 symptom is necessary for the diagnosis. ${ }^{1}$ SCAT3 contains a combination of clinical tests, including balance tests, and may be just as accurate as a computerized neuropsychological screening tool. ${ }^{15}$ There is also a new version of SCAT3 for children 5-12 years old, ChildSCAT3 (http://links.lww.com/JSM /A31). Both are new management tools, and information is limited about their performance characteristics. ${ }^{12}$

\section{Management}

In most countries, it is now standard practice for every concussed person to be removed from active participation and be evaluated by a medical doctor. This position was first advocated by the Canadian Academy of Sport Medicine in $2000^{16}$ and reaffirmed since then by experts at several international consensus conferences on concussion. ${ }^{1}$ The medical examination should occur within a reasonably short interval, preferably within a few hours after injury. This principle may not be achievable in remote regions, where responsibility should be delegated to other trained health care professionals.

Removal from play, work or school, and rest are the most important aspects of initial management. Based on the consensus of experts, "rest" now includes rest from both physical and cognitive activities. ${ }^{1}$ The inclusion of mental rest represents a major change in management. No known treatment other than rest has proven to be successful for early management.

Box 2: Spectrum of neurological and psychological disorders in concussion

- Acute concussion

- Second-impact syndrome or post-traumatic cerebral swelling

- Postconcussion syndrome (i.e., symptoms of concussion persist for weeks or months; more common after repeated concussions)

- Post-traumatic epilepsy (i.e., onset of epilepsy after head trauma)

- Psychosocial consequences (e.g., depression, anxiety, behavioural change, suicide)

- Chronic traumatic encephalopathy (i.e., a specific form of brain degeneration producing dementia or movement disorders; may appear many years after repeated concussions)

Source: McCrory et al.' 
Rest is the first of 6 steps in the return-toplay guidelines (Box 1), ${ }^{1}$ although the mechanism underlying its value is unknown. The recommended duration for the initial period of rest is $24-48$ hours. When all symptoms have completely disappeared in one step, the next step is initiated (Box 1). ${ }^{1}$ If exercise is introduced too soon, it may bring on new symptoms and worsen old ones. A physician must re-examine the patient after step 4 . The 6 graduated steps can be sport-specific with at least 24 hours between steps. Response to progressive exercise is a measure of brain recovery rather than a therapy, although it has been advocated by some as a therapy without definite proof. With 6 steps and at least 24 hours between steps, the earliest a concussed athlete can return to play is 1 week; most experts lengthen that period for children and youth. ${ }^{1}$ This is the most widely accepted form of management, but there is no trial evidence supporting this approach. This return-to-play protocol has replaced previous methods. ${ }^{1}$

If there is postconcussion syndrome (i.e., symptoms of concussion persisting for weeks or months), the athlete should not return to play. If postconcussion syndrome lasts several months, then permanent removal from collision sports should be considered. Such a decision is also recommended for persistent cognitive dysfunction; in this situation, formal, detailed neuropsychological testing is essential. The criteria for permanent removal from collision sports also include permanent neurologic deficits, movement disorders, and lesions seen on CT or MRI scans. ${ }^{17,18}$

Worrisome features of multiple concussions with respect to return to play include a high number of concussions, short interval between concussions, low severity of the forces that caused subsequent concussions, increasing time to recovery and young age of the athlete. ${ }^{1}$ Rest and avoidance of activities that precipitate symptoms are the only known approaches to management that accelerate recovery from concussion and that relieve most of the symptoms of repeated concussions.

\section{Consequences of concussions}

As mentioned previously, there are important early and late consequences of concussion (Box 2). ${ }^{1}$ The most important early consequence is the second-impact syndrome, which occurs when a second head injury occurs before the brain has fully recovered from a first injury such as a concussion. This syndrome is rare, and almost all cases have involved teenagers or young adults. The exact mechanism is unknown but is considered to be due to loss of autoregulation of cerebral vasculature resulting in cerebral swelling, raised intracranial pressure and subsequent brain herniation. The term "second-impact syndrome" is not accepted by all, but it is widely accepted that cerebral swelling can develop in concussed athletes after a latent period. ${ }^{3}$ Most of those affected have died, and survivors have had major neurologic deficits. This syndrome should be preventable by removing concussed athletes from play with no return until the 6-step protocol has been completed.

Early- or late-onset epilepsy can follow a concussion. Depression and anxiety are also possible. Post-traumatic depression and anxiety should be treated and typically respond to the same therapies as those for nontraumatic causes.

Chronic traumatic encephalopathy is posttraumatic degeneration of the brain after repeated concussions. Prominent clinical features include dementia, personality change, emotional disorders (especially depression) and movement disorders. The latent period after concussion is usually decades, but shorter latent periods have occurred in some cases. The brain degeneration is caused by abnormal deposition of hyperphosphorylated tau protein in neurons that leads to functional loss, neuronal destruction and neurofibrillary tangles. Although chronic traumatic encephalopathy shares several neuropathological features with

\section{Box 3: Prevention of concussions in collision sports}

\section{Primary prevention}

Prevent concussions from occurring:

- Implement "no checks to the head" rule in hockey

- Have players wear impact-softening shoulder and elbow pads

- Reduce head-to-head contact (i.e., football)

- Enforce rules

- Place emphasis on respect for the brain among players, including opponents' brains

\section{Secondary prevention}

Diagnose and manage concussions using best practices, such as the following:

- Every person suspected of having a concussion should be evaluated by a physician*

- Remove the concussed person from play

- Use 6-step return-to-play protocol to hasten recovery, and to prevent second-impact syndrome and cerebral swelling

\section{Tertiary prevention}

Prevent long-term complications such as chronic traumatic encephalopathy:

- Recommend permanent removal from play in selected cases, especially after repeated concussions

*In remote regions where a physician is not available, responsibility should be delegated to other trained health care professionals. 
Alzheimer disease, it appears to be a distinct entity. ${ }^{4}$ Chronic traumatic encephalopathy was first described in boxers, but it has since been identified in other athletes with repeated concussions, including football, hockey and soccer players. $^{4,19}$

\section{Prevention}

Box 3 shows the 3 phases of injury prevention for concussions, and physicians should be active in all phases. Physicians can promote primary prevention by advocacy for enhanced legislation, such as exists in many US states, to detect and prevent head injuries in sport and recreation. This may include mandatory education about concussions and best practices for management targeted to children and youth in team collision sports and their parents, coaches, trainers and referees. Helmets prevent catastrophic brain injuries such as cerebral lacerations and intracranial hematomas; however, helmets that are cur-

\section{Box 4: Practice points on the diagnosis and management of concussion}

Unless specified otherwise, these points are based on the International Consensus Conference on Concussion in Sport ${ }^{1}$ and represent changes in our understanding of concussion over the past decade

\section{Definition and mechanism}

- Any alteration of mental functioning after a blow to the head or body is a concussion

- Concussion is a brain injury; "dings" and "seeing stars" are symptoms of a concussion

- The definition of concussion does not include loss of consciousness (95\% of concussions do not cause a loss of consciousness)

- A direct blow to the head is not necessary to produce a concussion; whiplash from a blow to the body can cause it

- There are some similarities between a concussion and a brain injury from a military blast

- Previous systems for grading a concussion should be discarded because they are not evidence-based

\section{Risk factors}

- There may be a genetic susceptibility to concussion"11

- The young brain concusses more easily than the adult brain ${ }^{8}$

- Females may be more susceptible to concussion than males ${ }^{10}$

\section{Diagnosis}

- The presence of only 1 persisting symptom (e.g., headache, dizziness, nausea and imbalance) qualifies as a concussion

- Routine computed tomography and magnetic resonance imaging scans almost always appear normal in concussion

- Only a physician is qualified to make the diagnosis of concussion*

- SCAT3 (for youth 13 and older and adults) and the new ChildSCAT3 (for children 5-12 years old) are useful evaluation tools for physicians

\section{Consequences}

- The next concussion generally occurs after less force than the previous one ${ }^{19}$

- Recovery time after the next concussion is generally longer than after the previous one ${ }^{19}$

- Second-impact syndrome and cerebral swelling after concussion are rare, potentially fatal consequences of concussion ${ }^{3}$

- Punch drunk syndrome is now known as chronic traumatic encephalopathy

- Repeated concussions in any sport or activity, not just boxing, can cause chronic traumatic encephalopathy

- Repeated concussions can cause depression and anxiety unrelated to disappointment about not returning to play ${ }^{19}$

\section{Management}

- Every concussed person should see a physician*

- The young brain takes longer to recover from concussion than the adult brain ${ }^{8}$

- The only treatment agreed upon for the early stage of concussion is rest; in the later stages, treatment for depression and anxiety are indicated

- Rest now includes rest from both physical and cognitive activities

- A 6-step return-to-play protocol is recommended and is based on graduated exercise

- A physician should assess the patient again after step 4 (see Box 1)

- Exercise may bring on new symptoms and worsen old symptoms; during recovery, patients should stay below the activity threshold for eliciting symptoms of concussion

- Return to school or work should be on a graduated basis for physical and cognitive activities

- Baseline computerized neuropsychological screening has not been proven to be essential for the management of concussion ${ }^{1,14}$

- Formal neuropsychological testing is valuable for assessing the need for permanent removal from collision sports, and to aid return to school or work when psychological deficits persist

SCAT3 = third edition of the Sport Concussion Assessment Tool, available at http://links.Iww.com/JSM/A30; ChildSCAT3 is available at http://links.Iww.com/JSM/A31. *In remote regions where a physician is not available, responsibility should be delegated to other trained health care professionals. 
rently available do not prevent all concussions because they do not eliminate rotational acceleration of the brain. ${ }^{5}$

Physicians should also play major roles in secondary prevention by facilitating recovery from concussion and by early diagnosis of posttraumatic cerebral swelling. Tertiary prevention involves recommendations by physicians to terminate further participation in collision sports to prevent or diminish the severity of chronic traumatic encephalopathy.

Educating the public about concussion is an important component of primary, secondary and tertiary prevention. Everyone who is engaged in sports should be aware of the importance of recognizing concussion. However, as stated earlier, the responsibility for diagnosing concussion rests with the physician, or a trained delegate in remote regions.

Box 4 outlines practice points about the diagnosis and management of concussion that have been updated over the past decade. ${ }^{1,3,4,8,10,11,14,19}$ Resources for clinicians are available in Box 5.

\section{Box 5: Resources for clinicians}

- ThinkFirst Canada (www.thinkfirst.ca): Information sheets on concussion for physicians and others, and a full description of the 6-step return-to-play protocol

- Sports Concussion Assessment Tool, third edition (SCAT3), for youth 13 years and older and adults (http://links.Iww.com/JSM/A30); and the new ChildSCAT3, for children 5-12 years old (http://links.Iww.com/JSM/A31)

- US Centers for Disease Control and Prevention (www.cdc.gov/concussion/sports /index.html): Tips on the management of concussion by physicians in the "Heads Up" program

- Canadian Medical Association policy statement on head injury and sport (http://policybase.cma .ca/dbtw-wpd/Policypdf/PD11-10.pdf)

- Canadian Paediatric Society position statement on the evaluation and management of children and adolescents with sports-related concussion (www.cps.ca/english/statements /HAL/HAL12-01.htm)

\section{References}

1. McCrory P, Meeuwisse WH, Aubry M, et al. Consensus statement on concussion in sport: the 4th International Conference on Concussion in Sport held in Zurich, November 2012. Br J Sports Med 2013;47:250-8.

2. Tator CH. Let's standardize the definition of concussion and get reliable incidence data. Can J Neurol Sci 2009;36:405-6.

3. McCrory P, Davis G, Makdissi M. Second impact syndrome or cerebral swelling after sporting head injury. Curr Sports Med Rep 2012;11:21-3.

4. McKee AC, Cantu RC, Nowinski CJ, et al. Chronic traumatic encephalopathy in athletes: progressive tauopathy after repetitive head injury. J Neuropathol Exp Neurol 2009;68:709-35.

5. Hoshizaki TB, Brien SE. The science and design of head protection in sport. Neurosurgery 2004;55:956-66; discussion 966-7.

6. Vagnozzi R, Tavazzi B, Signoretti S, et al. Temporal window of metabolic brain vulnerability to concussions: mitochondrialrelated impairment-part I. Neurosurgery 2007;61:379-88; discussion 388-9.

7. Hynes LM, Dickey JP. Is there a relationship between whiplashassociated disorders and concussion in hockey? A preliminary study. Brain Inj 2006;20:179-88.

8. Baillargeon A, Lassonde M, Leclerc S, et al. Neuropsychologi$\mathrm{cal}$ and neurophysiological assessment of sport concussion in children, adolescents and adults. Brain Inj 2012;26:211-20.

9. Guskiewicz KM, McCrea M, Marshall SW, et al. Cumulative effects associated with recurrent concussion in collegiate football players: the NCAA Concussion Study. JAMA 2003;290:2549-55.

10. Gessel LM, Fields SK, Collins CL, et al. Concussions among United States high school and collegiate athletes. J Athl Train 2007;42:495-503.

11. Kristman VL, Tator CH, Kreiger N, et al. Does the apolipoprotein epsilon 4 allele predispose varsity athletes to concussion? A prospective cohort study. Clin J Sport Med 2008;18:322-8.

12. Echlin PS, Tator $\mathrm{CH}$, Cusimano MD, et al. A prospective study of physician-observed concussions during junior ice hockey: implications for incidence rates. Neurosurg Focus 2010;29:E4.

13. Ackery A, Provvidenza C, Tator $\mathrm{CH}$. Concussion in hockey: compliance with return to play advice and follow-up status. Can J Neurol Sci 2009;36:207-12.

14. Randolph C. Baseline neuropsychological testing in managing sport-related concussion: Does it modify risk? Curr Sports Med Rep 2011;10:21-6.

15. Echlin PS, Tator $\mathrm{CH}$, Cusimano MD, et al. Return to play after an initial or recurrent concussion in a prospective study of physician-observed junior ice hockey concussions: implications for return to play after a concussion. Neurosurg Focus 2010;29:E5.

16. Guidelines for assessment and management of sport-related concussion. Canadian Academy of Sport Medicine Concussion Committee. Clin J Sport Med 2000;10:209-211.

17. Tator CH. Spinal cord and brain injuries in ice hockey. In: Bailes JE, Day AL, editors. Neurological sports medicine: a guide for physicians and athletic trainers. Rolling Meadows (IL): American Association of Neurological Surgeons; 2001:261-71.

18. Cantu RC, Register-Mihalik JK. Considerations for return-toplay and retirement decisions after concussion. PM R 2011;3(10 Suppl 2):S440-4.

19. Martland HS. Punch drunk. JAMA 1928;92:1103-7.

20. Guskiewicz KM, Marshall SW, Bailes J, et al. Recurrent concussion and risk of depression in retired professional football players. Med Sci Sports Exerc 2007;39:903-9.

Affiliations: The Division of Neurosurgery, University of Toronto; the Canadian Sports Concussion Project, Krembil Neuroscience Centre, Toronto Western Hospital; and ThinkFirst and Parachute Canada, Toronto, Ont. 